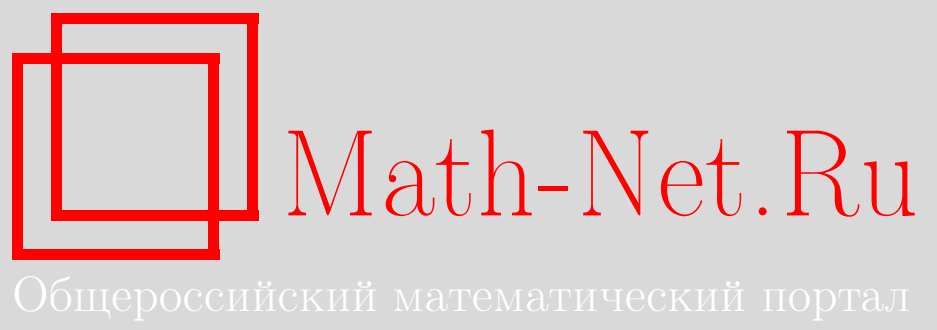

А. А. Коньков, О поведении решений квазилинейных эллиптических неравенств, содержащих члены с младшими производными, Матем. заметки, 1998, том 64, выпуск 6, 946949

DOI: https://doi.org/10.4213/mzm1474 
Использование Общероссийского математического портала Math-Net.Ru подразумевает, что вы прочитали и согласны с пользовательским соглашением http://www. mathnet.ru/rus/agreement

Параметры загрузки:

IP: 54.197 .130 .99

26 апреля 2023 г., 10:07:21

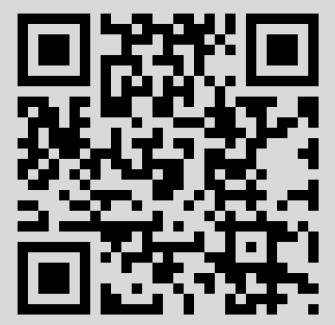




\section{О ПОВЕДЕНИИ РЕШЕНИЙ \\ КВАЗИЛИНЕЙНЫХ ЭЛЛИПТИЧЕСКИХ НЕРАВЕНСТВ, СОДЕР ЖАЩИХ ЧЛЕНЫ С МЛАДШИМИ ПРОИЗВОДНЫМИ}

\section{А. А. Коньков}

Предположим, что $\Omega$ - непустое открытое подмножество $\mathbb{R}^{n}, n \geqslant 2, \mathscr{L}$ - дифференциальный оператор вида

$$
\mathscr{L}=\sum_{i, j=1}^{n} a_{i j}(x) \frac{\partial^{2}}{\partial x_{i} \partial x_{j}}+\sum_{i=1}^{n} b_{i}(x) \frac{\partial}{\partial x_{i}}
$$

где $a_{i j} \equiv a_{j i}$, и для некоторого вещественного числа $C>0$ при всех $x=\left(x_{1}, \ldots, x_{n}\right) \in \Omega$ и $\xi=\left(\xi_{1}, \ldots, \xi_{n}\right) \in \mathbb{R}^{n}$ выполнено условие эллиптичности

$$
0<\sum_{i, j=1}^{n} a_{i j}(x) \xi_{i} \xi_{j} \leqslant C|\xi|^{2}
$$

а коэффициенты при младших производных $b_{i}$ являются локально ограниченньпи функциями.

Под $Q_{r}, B_{r}$ и $S_{r}$ подразумеваем соответственно открытый, замкнутый шары и сферу радиуса $r \geqslant 0$ с центром в начале координат, а именно: $Q_{r}=\{x:|x|<r\}, B_{r}=\{x:|x| \leqslant r\}$, $S_{r}=\{x:|x|=r\}$.

Вопросы, рассматриваемые в этой статье, ранее исследовались в работах [1]-[6].

Работа выполнена при финансовой поддержке программы "Ведущие научные школы", грант № 96-15-96177. 
Будем изучать (понимаемые в классическом смысле) неотрицательные решения неравенств

$$
\mathscr{L} u \geqslant F(x, u),
$$

где функция $F: \Omega \times[0, \infty) \rightarrow[0, \infty)$ монотонно не убьвает по второму аргументу.

Для краткости обозначим

$$
M(r ; u)=\sup _{S_{r} \cap \Omega} u .
$$

TeOpema 1. Пусть $0 \leqslant R_{0}<R \leqslant \infty, S_{R_{0}} \cap \bar{\Omega} \neq \varnothing$ u nyсmь $\sigma>1, k_{1}>0, k_{2}>0-$ некоторые вещественные числа, а $b:\left(R_{0}, \infty\right) \rightarrow[0, \infty)$ u $f:\left(R_{0}, \infty\right) \times(0, \infty) \rightarrow[0, \infty)-$ измеримые функции такие, что

$$
\begin{gathered}
b(r) \geqslant \sup _{x \in \Omega \cap Q_{r \sigma} \backslash\left(B_{r / \sigma} \cup B_{R_{0}}\right)} \sum_{i=1}^{n}\left|b_{i}(x)\right| \quad \text { для всех } r \in\left(R_{0}, \infty\right), \\
f(r, t) \leqslant \inf _{x \in \Omega \cap Q_{r \sigma} \backslash\left(B_{r / \sigma} \cup B_{R_{0}}\right)} F(x, t) \quad \partial \Omega \text { в всех } \quad r \in\left(R_{0}, \infty\right), \quad t \in(0, \infty),
\end{gathered}
$$

причем функция $f$ монотонно не убывает по второму аргументу и полунепрерывна слева $\left(\right.$ m.e. $f(r, t-0)=f(r, t)$ для всех $\left.r \in\left(R_{0}, \infty\right), t \in(0, \infty)\right)$.

Предположим, что и является неотричательным решением неравенства (2) в области $\Omega \cap Q_{R} \backslash B_{R_{0}}$ и удовлетворяет условиям

$$
\begin{gathered}
M\left(R_{0}+0 ; u\right)>0,\left.\quad u\right|_{Q_{R} \cap \partial \Omega \backslash B_{R_{0}}}=0, \\
M\left(r_{1} ; u\right) \leqslant M\left(r_{2} ; u\right) \quad n p u \quad r_{1} \leqslant r_{2} .
\end{gathered}
$$

Тогда найдутся константы $\alpha>0, \beta>0$, зависящие только от $n, \sigma, k_{1}, k_{2}$, а также от постоянной $C$ из неравенства (1), такие, что существует определенное на всем промехутке $\left[R_{0}, R\right)$ решение задачи Коши

$$
\begin{gathered}
m^{\prime \prime}+\left(\frac{1+k_{1}}{r}+k_{2} b(r)\right) m^{\prime}=\alpha f(r, \beta m), \\
m\left(R_{0}\right)=M\left(R_{0}+0 ; u\right), \quad m^{\prime}\left(R_{0}\right)=0,
\end{gathered}
$$

для которого при всех $r \in\left(R_{0}, R\right)$ выполнена оченка $M(r ; u) \geqslant m(r)$.

ЗАмЕчАниЕ. Если $n \geqslant 3$, то, полагая в теореме $1 k_{1}=n-2, k_{2}=1$, получим в левой части уравнения (5) радиальную часть оператора $\Delta+b(|x|) \nabla|x| \cdot \nabla$.

Теорема 1 является следствием более общего утверждения, которое мы и приводим ниже.

Теорема 2. Пусть выполнены предположения теоремы 1. Тогда найдутся константы $\alpha>0, \beta>0$, зависящие только от $n, \sigma, k_{1}, k_{2}$ u $C$, такие, что для всякого $r \in\left(R_{0}, R\right)$

$$
M(r ; u)-M\left(R_{0}+0 ; u\right) \geqslant \alpha \int_{R_{0}}^{r} K(r, \xi) f(\xi, \beta M(\xi ; u)) d \xi
$$

$2 \partial e$

$$
K(r, \xi)=\int_{\xi}^{r} d \rho\left(\frac{\xi}{\rho}\right)^{1+k_{1}} \exp \left(-k_{2} \int_{\xi}^{\rho} b(s) d s\right) .
$$

Далее, если не оговорено противное, мы будем считать, что $\sigma>1$ и $R_{0}>0$ - фиксированные вещественные числа, $S_{R_{0}} \cap \bar{\Omega} \neq \varnothing$, при этом для измеримой функции $b:\left(R_{0}, \infty\right) \rightarrow[0, \infty)$ выполнено соотношение (3). Будем также считать, что $p:\left(R_{0}, \infty\right) \rightarrow[0, \infty)$ и $g:(0, \infty) \rightarrow(0, \infty)$ - измеримые функции такие, что для всех $r \in\left(R_{0}, \infty\right)$ и $t \in(0, \infty)$

$$
p(r) g(t) \leqslant \inf _{x \in \Omega \cap Q_{r \sigma} \backslash\left(B_{r / \sigma} \cup B_{R_{0}}\right)} F(x, t),
$$

причем $g\left(t_{1}\right) \leqslant g\left(t_{2}\right)$, если $t_{1} \leqslant t_{2}$. 
Tеорема 3. Пусть для некоторых $k_{1}, k_{2} \in(0, \infty)$

$$
\int_{R_{0}}^{\infty} d r p(r) \int_{r}^{\infty} d s\left(\frac{r}{s}\right)^{1+k_{1}} \exp \left(-k_{2} \int_{r}^{s} b(\rho) d \rho\right)=\infty
$$

Тогда $е с л u$

$$
\int_{1}^{\infty}(g(t) t)^{-1 / 2} d t<\infty
$$

то в области $\Omega \backslash B_{R_{0}}$ всякое неотричательное решение неравенства (2), удовлетворяющ,е условию (4) и такое, что

$$
\left.u\right|_{\partial \Omega \backslash B_{R_{0}}}=0,
$$

тохдественно равно 0.

ТеОРема 4. Пусть выполнены соотношения (6) и (7), где $k_{1}, k_{2} \in(0, \infty)$. Тогда если в дополнение к сказанному функиия $F(x, t)$ является выпуклой по $t$, m.e.

$$
F\left(x, \varkappa t_{1}+(1-\varkappa) t_{2}\right) \leqslant \varkappa F\left(x, t_{1}\right)+(1-\varkappa) F\left(x, t_{2}\right), \quad x \in \Omega, \quad t_{1}, t_{2} \in(0, \infty), \quad \varkappa \in[0,1],
$$

то решение задачи Дирихле

$$
\mathscr{L} u=F(x,|u|) \operatorname{sign} u \quad{ }_{\theta} \Omega,\left.\quad u\right|_{\partial \Omega}=\psi,
$$

әде $\psi \in C(\partial \Omega)$, единственно (при этом никаких условий на бесконечности не предполаzaemcr).

Предположим, что $q: \Omega \rightarrow[0, \infty)$ и $p:\left(R_{0}, \infty\right) \rightarrow[0, \infty)$ - измеримые функции такие, что

$$
p(r) \leqslant \inf _{x \in \Omega \cap Q_{r \sigma} \backslash\left(B_{r / \sigma} \cup B_{R_{0}}\right)} q(x) \quad \text { для всех } r \in\left(R_{0}, \infty\right),
$$

где $R_{0}>0, S_{R_{0}} \cap \bar{\Omega} \neq \varnothing$. По-прежнему считаем, что для измеримой функции $b:\left(R_{0}, \infty\right) \rightarrow[0, \infty)$ справедливо соотношение (3).

СледСтвиЕ. Пусть $\lambda>1, u$ для некоторых $k_{1}, k_{2} \in(0, \infty)$ выполнено условие (6). Тогда в области $\Omega \backslash B_{R_{0}}$ всякое неотрицательное решение неравенства

$$
\mathscr{L} u \geqslant q(x) u^{\lambda}
$$

удовлетворяющее условиям (4), (8), тождественно равно 0. Более того, в этом случае решение задачи Дирихле

$$
\mathscr{L} u=q(x)|u|^{\lambda} \operatorname{sign} u \quad \text { в } \Omega,\left.\quad u\right|_{\partial \Omega}=\psi,
$$

әде $\psi \in C(\partial \Omega)$, единственно.

Во всех приведенных ниже примерах $\lambda>1$.

ПримеР 1. Допустим, что коэффициенты при младшшх производных оператора $\mathscr{L}$ имеют в окрестности бесконечно удаленной точки не более чем степенной рост:

$$
\sum_{i=1}^{n}\left|b_{i}(x)\right| \leqslant A|x|^{k}
$$

где $A$ и $k$ - некоторые вещественные числа. Тогда условие (6) при $k>-1$ принимает вид

$$
\int_{R_{0}}^{\infty} r^{-k} p(r) d r=\infty
$$


а при $k \leqslant-1-$

$$
\int_{R_{0}}^{\infty} r p(r) d r=\infty
$$

В частности, если $p(r) \sim r^{l}$ при $r \rightarrow \infty$, то при $k>-1$ и $l \geqslant k-1$ либо при $k \leqslant-1$ и $l \geqslant-2$ в области $\Omega \backslash B_{R_{0}}$ любое неотрицателное решение неравенства (9), удовлетворяющее условиям (4), (8), тождественно равно 0; кроме того, имеет место единственность решения задачи Дирихле (10).

В то же время, в области $\mathbb{R}^{n} \backslash B_{R_{0}}$ несложно построить положительное решение неравенства

$$
\Delta u+|x|^{k} \nabla|x| \cdot \nabla u \geqslant p(|x|) u^{\lambda},
$$

где $p(r) \sim r^{l}$ при $r \rightarrow \infty$, удовлетворяющее условию (4), а именно:

$$
u(x)= \begin{cases}|x|^{(k-l-1) /(\lambda-1)}, & \text { если } k>-1 \text { и } l<k-1, \\ |x|^{(2+l) /(1-\lambda)}, & \text { если } k \leqslant-1 \text { и } l<-2 .\end{cases}
$$

ПримеР 2. Пусть в окрестности бесконечно удаленной точки

$$
\sum_{i=1}^{n}\left|b_{i}(x)\right| \leqslant A|x|^{k} \ln ^{l}|x|,
$$

где $A, k, l$ - некоторые вешественные числа, причем $k>-1$ либо $k=-1$ и $l>0$. Тогда соотношение (6) принимает вид

$$
\int_{R_{0}}^{\infty} r^{-k} \ln ^{-l} r p(r) d r=\infty
$$

В частности, если $p(r) \sim r^{k-1}$ при $r \rightarrow \infty$, то в случае $l \leqslant 1$ в области $\Omega \backslash B_{R_{0}}$ любое неотрицательное решение неравенства (9), удовлетворяющее условиям (4), (8), тождественно равно 0 ; кроме того, имеет место единственность решения задачи Дирихле (10).

В то же время, для $l>1$ несложно убедиться в том, что функция $u(x)=(\ln |x|)^{(l-1) /(\lambda-1)}$ является в области $\mathbb{R}^{n} \backslash B_{R_{0}}$ положительным решением неравенства

$$
\Delta u+|x|^{k} \ln ^{l}|x| \nabla|x| \cdot \nabla u \geqslant p(|x|) u^{\lambda}
$$

где $p(r) \sim r^{k-1}$ при $r \rightarrow \infty$, удовлетворяющим соотношению (4).

Примеры 1, 2 свидетельствуют о точности условия (6).

\section{СПИСОК ЦИТИРОВАННОЙ ЛИТЕРАТУРЫ}

1. Keller J. B. // Comm. Pure Appl. Math. 1957. V. 10. №4. P. 503-510. 2. Osserman R. // Pacific J. Math. 1957. V. 7. № 4. P. 1641-1647. 3. Vazquez J. L. // Nonlinear Anal. 1981. V. 5. Р. 95-103. 4. Кондратьев В.А., Ландис Е. М. // Матем. сб. 1988. Т. 135 (177). № 3. С. 346-360. 5. Кондратьев В. А., Коньков А. А. // Матем. сб. 1994. Т. 185. №9. С. 81-94. 6. Kon'kov A. A. // Russian J. Math. Phys. 1997. V. 5. № 1. P. 119-122. 